\title{
The Application of the Rome IV Criteria to Functional Esophagogastroduodenal Disorders in Asia
}

\author{
Hidekazu Suzuki \\ Medical Education Center, Keio University School of Medicine, Tokyo, Japan
}

The Rome criteria were amended as Rome IV. For functional esophageal disorders, the exclusion criteria have been more specifically revised based on further understanding of other esophageal disorders, including eosinophilic esophagitis and spastic and hypercontractile motor disorders. Another revised point is the more restrictive definition of gastroesophageal reflux disease, indicating that sensitivity to a physiological reflux burden may be placed more firmly within the functional group. For functional dyspepsia (FD), only minor changes were introduced, mainly to improve specificity. Among the major symptoms of FD, not only postprandial fullness, but also epigastric pain, epigastric burning, and early satiation should be "bothersome." Investigation on the effect of meal ingestion on symptom generation has indicated that not only postprandial fullness and early satiety, but also epigastric pain, epigastric burning sensation and nausea (not vomiting) may increase after meals. Helicobacter pylori infection is considered a possible cause of dyspepsia if successful eradication leads to sustained resolution of symptoms for more than 6 months, and such status can be termed as " $H$. pylori-associated dyspepsia." Prompt esophagogastroduodenoscopy and H. pylori testing and treatment would be more beneficial, especially in Asia, which has a high prevalence of gastric cancer. Acotiamide, tandospirone, and rikkunshito are the newly listed as treatment options for FD. For further therapeutic development, clinical studies based on the strict Rome IV criteria should be performed.

(J Neurogastroenterol Motil 2017;23:325-333)

Key Words

Belching; Dyspepsia; Globus; Heartburn; Nausea

\section{Introduction}

The Rome criteria were amended as the Rome IV criteria, launched at the site of Digestive Disease Week (DDW2016) in San Diego, California, USA, on May 21-25, 2016. ${ }^{1}$ The Rome IV Education Materials for Functional Gastrointestinal Disorders is composed of 6 printed books and online materials. The new Rome IV publications have been updated since Rome III in 2006, with new chapters, references, diagnoses, and graphics, and included the work of more than 120 medical researchers and clinicians from all over the world. Rome IV publications and educational materials are the summary of 5 years of work-ups based on decades of research (2007-2016). The new Rome IV series includes (1) Functional Gastrointestinal Disorders - Disorders of Gut-Brain Interaction (vol. 1 \& 2), (2) Multidimensional Clinical Profile for Functional Gastrointestinal Disorders: MDCP, (3) Diagnostic Algorithms for Common GI Symptoms, (4) Functional Gastrointestinal Disorders for Primary Care and Non-GI Clinicians, (5) Pediatric Functional Gastrointestinal Disorders - Disorders of Gut-Brain Interaction,

Received: February 15, 2017 Revised: None Accepted: June 7, 2017

(c) This is an Open Access article distributed under the terms of the Creative Commons Attribution Non-Commercial License (http://creativecommons. org/licenses/by-nc/4.0) which permits unrestricted non-commercial use, distribution, and reproduction in any medium, provided the original work is properly cited.

*Correspondence: Hidekazu Suzuki, MD, PhD, FACG, AGAF, RFF

Medical Education Center, Keio University School of Medicine, 35 Shinanomachi, Shinjuku-ku, Tokyo 160-8582, Japan Tel: +81-3-5363-3914, Fax: +81-3-5363-3967, E-mail: hsuzuki.a6@keio.jp 
and (6) Diagnostic Questionnaires and Tables for Investigators and Clinicians. ${ }^{1}$

Among them, criteria for upper gastrointestinal (GI) lesions have been developed for functional esophageal disorders ${ }^{3}$ and functional gastroduodenal disorders. ${ }^{4}$

\section{Functional Esophageal Disorders}

In the Rome IV section on functional esophageal disorders, ${ }^{4}$ the exclusion criteria have been more specifically revised based on greater and updated understanding of esophageal disorders, including eosinophilic esophagitis (EoE) and structural esophageal motor disorders. In contrast, ineffective esophageal motility and fragmented peristalsis are not included in the present exclusion criteria because these motor phenotypes can be encountered in asymptomatic cohorts and seem to generate symptoms secondary to gastroesophageal reflux disease (GERD), visceral hypersensitivity, and hypervigilance. Symptoms derived from esophageal mechanical obstruction such as esophagogastric junctional (EGJ) outflow obstruction should be strictly excluded by endoscopic ultrasound or contrast radiology because these might be related to achalasia in evolution or to a subtle mechanical obstruction. To exclude EoE, upper GI endoscopy (linear furrow, etc) and/or mucosal biopsy is recommended. Another revised point is the more restrictive definition of GERD, indicating that sensitivity to a physiological reflux burden may be placed more firmly within functional disorders. Although patients with symptom-reflux correlation with physiological reflux episodes may respond to anti-secretory agents such as proton pump inhibitors (PPIs; recently in Japan, potassium-competitive acid blocker [P-CAB], vonoprazan, has been launched ${ }^{6}$ ) or histamine $\mathrm{H} 2$ receptor antagonists $\left(\mathrm{H}_{2} \mathrm{RA}\right)$ treatment, the current understanding of visceral hypersensitivity and mechanisms of sensitization indicates that these are functional disorders. In Rome IV, symptoms of erosive esophagitis (reflux esophagitis) are dominated by extraordinary acid exposure, whereas symptoms of functional heartburn are dominated by visceral hypersensitivity. Non-erosive reflux disease (NERD) and reflux hypersensitivity are intermediate disease entities classified between erosive esophagitis (reflux esophagitis) and functional heartburn. Ambulatory $\mathrm{pH}$ monitoring and highresolution manometry are not always available in every hospital, but resistance to a PPI trial for reflux symptoms remains an indication for second-stage evaluation. Peripheral or central hypersensitivity in viscera is a potentially unifying pathophysiological concept in functional heartburn and reflux hypersensitivity. In Japan, vonoprazan, a novel and potent first-in-class P-CAB, was launched ${ }^{5,6}$ and now is expected to prove useful even in the treatment of functional esophageal disorders triggered by acid hypersensitivity. ${ }^{7}$

Functional esophageal disorders include functional chest pain (A1), functional heartburn (A2), reflux hypersensitivity (A3), globus (A4), and functional dysphagia (A5) in the Rome IV edition. Among these 5 disease categories, functional chest pain was previously named as functional chest pain that is presumed to be originated from the esophagus in the Rome III edition, and reflux hypersensitivity has been newly added to the present Rome IV edition.

\section{Functional Chest Pain}

As mentioned above, functional chest pain was described as functional chest pain of presumed esophageal origin in Rome III. Although most of the previous studies assessed non-cardiac chest pain (NCCP) as a presumed representative of functional chest pain, in the newly revised Rome IV, functional chest pain is not equal to NCCP, but is clearly defined as a part of the broad umbrella disease entity of NCCP. In other words, NCCP also includes other esophageal disorders such as GERD, erosive esophagitis, and esophageal motor disorders aside from pure functional chest pain. According to the epidemiological study by Fass and Dickman, ${ }^{8}$ among patients with NCCP, 50-60\% had GERD, 15-18\% had esophageal motor disorders, and $32-35 \%$ had true functional chest pain. In the Rome IV diagnostic criteria, frequency of symptoms is at least once a week and exclusions of the other esophageal disease processes including GERD, EoE, and major structural or organic esophageal motility disorders such as achalasia, EGJ outflow obstruction, diffuse esophageal spasm, jackhammer esophagus, and absent peristalsis are definitely excluded.

\section{Functional Heartburn}

In Rome III, the acid-sensitive esophagus previously included in functional heartburn in Rome II was shifted to a part of the group of NERD. ${ }^{9}$ Such acid-sensitive esophagus is defined as an independent novel disease entity as reflux hypersensitivity as was listed in A3 of Rome IV. Then, the definition of true functional heartburn is further narrowed by confirming a lack of conclusive evidence for GERD, no evidence of a symptom-reflux correlation, and a negative response to anti-secretory agents such as PPI or P$\mathrm{CAB}$. The diagnostic criteria of functional heartburn are defined as frequency of symptoms of at least twice a week, no symptom relief by PPI, and exclusion of the other esophageal diseases including 
GERD, EoE, and major esophageal motor disorders, as mentioned in the section of functional chest pain.

\section{Reflux Hypersensitivity}

This disease entity is a newcomer to the Rome IV. The Rome III criteria of functional esophageal disorders had a controversy in their expansion of the definition of NERD to include cohorts with normal acid exposure to esophagus but with positive refluxsymptom correlation. In line with this controversy, the Rome committee developed a novel entity between NERD and functional heartburn. ${ }^{3}$ Since symptom-generation mechanisms in patients with acid-sensitive esophagus are mainly supposed to be enhanced sensitivity, a disease termed as "reflux hypersensitivity" was newly introduced to Rome IV. This disease entity is characterized as physiological (normal) acid reflux that might include esophageal mucosal histological changes including dilated intercellular spaces, basal cell thickness, and papillary elongation, as compared to functional heartburn.

In the Asian setting, to investigate the pathophysiology of functional heartburn, Tamura et $\mathrm{al}^{10}$ examined 111 patients with PPI-refractory non-erosive GERD by using intraesophageal pressure testing and 24-hour multichannel intraluminal impedance$\mathrm{pH}(24 \mathrm{MII}-\mathrm{pH})$ testing. In this study, they excluded 33 patients with esophageal motility disorders, while 22 patients with abnormal esophageal acid exposure time and 34 patients with hypersensitive esophagus were included in the endoscopy-negative reflux disease group. In this report published in 2015, the concept of a hypersensitive esophagus, that was newly introduced as an independent disease entity in the Rome IV criteria, has been clearly recognized in Japan, but with apparent separation of hypersensitive esophagus from NERD.

\section{Globus}

This disease is characterized as a persistent or intermittent, non-painful sensation of a foreign body or lump in the throating area with no structural esophageal lesion. These symptoms occur between meals without dysphagia or odynophagia as well as without gastric inlet patch (congenital ectopic gastric mucosa) in the proximal esophagus. In Rome IV, the gastric inlet patch is excluded owing to the recent insights on this lesion for globus symptom generation. In addition, a recent advance in the concerted effort to support endoscopic evaluation of the oropharynx is included in the diagnosis.

\section{Functional Dysphagia}

The Rome classification defines functional dysphagia as a sensation of abnormal bolus passage through the esophageal body without structural, esophageal mucosal, or motor disorders to explain the symptoms. In Rome IV, the exclusion criteria are definitely defined in terms of EoE, in which dysphagia can occur even without overt structural lesions, as well as major motor disorders that can be associated with abnormal bolus transit leading to dysphasia. ${ }^{3}$

Despite high prevalence rates and increasing medical concern, functional esophageal disorders such as functional chest pain, functional heartburn, reflux hypersensitivity, globus and functional dysphagia have not yet been well investigated. Further high-technology approaches to measure the gastroesophageal reflux events, esophageal motility, and esophageal sensation to validate these diagnostic criteria should be explored.

\section{Functional Gastroduodenal Disorders}

In Rome IV, although functional gastroduodenal disorders were again classified into 4 categories, as in Rome III: functional dyspepsia (FD) composed of postprandial distress syndrome (PDS) and epigastric pain syndrome (EPS), belching disorders composed of excessive gastric and supragastric belching, chronic nausea and vomiting disorders composed of chronic nausea vomiting syndrome (CNVS), cyclic vomiting syndrome (CVS), and the newly listed "cannabinoid hyperemesis syndrome" (CHS), and rumination syndrome, there are some changes in each component, especially for the sections for belching disorders and chronic nausea and vomiting disorders.

\section{Functional Dyspepsia}

FD is a condition of impaired digestion defined as the presence of dyspeptic symptoms as PDS mainly composed of early satiation or postprandial fullness and EPS, mainly composed of pain or burning in the epigastric region in the absence of an organic disease that is likely to explain these symptoms. ${ }^{11}$ In the Rome IV section on $\mathrm{FD},{ }^{5}$ only minor changes were introduced as compared to the previous Rome III criteria, ${ }^{11}$ mainly to improve the specificity of the definition (Table). Among the major symptoms of FD, not only postprandial fullness, but also epigastric pain, epigastric burning, and early satiation should be "bothersome" symptoms. In line with Rome III, FD again includes 2 subcategories (syndromes): PDS 
Table. Comparison of Rome IV with Rome III Criteria in Terms of Functional Gastroduodenal Disorders

\begin{tabular}{|c|c|}
\hline Rome IV & Rome III \\
\hline B. Functional gastroduodenal disorders & B. Functional gastroduodenal disorders \\
\hline B1. Functional dyspepsia & B1. Functional dyspepsia \\
\hline B1a. Postprandial distress syndrome & B1a. Postprandial distress syndrome \\
\hline B1b. Epigastric pain syndrome & B1b. Epigastric pain syndrome \\
\hline B2. Belching disorders & B2. Belching disorders \\
\hline B2a: Excessive supragastric belching (from esophagus) & B2a. Aerophagia \\
\hline B2b: Excessive gastric belching (from stomach) & B2b. Unspecified excessive belching \\
\hline B3. Nausea and vomiting disorders & B3. Nausea and vomiting disorders \\
\hline B3a: Chronic nausea and vomiting syndrome & B3a. Chronic idiopathic nausea \\
\hline B3b: Cyclic vomiting syndrome & B3b. Functional vomiting \\
\hline B3c: Cannabinoid hyperemesis syndrome & B3c. Cyclic vomiting syndrome \\
\hline B4: Rumination syndrome & B4. Rumination syndrome in adults \\
\hline B1. Functional dyspepsia & B1. Functional dyspepsia \\
\hline Diagnostic criteria & Must include \\
\hline 1. One or more of: & 1. One or more of: \\
\hline a. Bothersome postprandial fullness & a. Bothersome postprandial fullness \\
\hline b. Bothersome early satiation & b. Early satiation \\
\hline c. Bothersome epigastric pain & c. Epigastric pain \\
\hline d. Bothersome epigastric burning & d. Epigastric burning \\
\hline AND & AND \\
\hline $\begin{array}{l}\text { No evidence of structural disease (including at upper endoscopy) } \\
\text { that is likely to explain the symptoms }\end{array}$ & $\begin{array}{l}\text { No evidence of structural disease (including at upper endoscopy) } \\
\text { that is likely to explain the symptoms }\end{array}$ \\
\hline \multicolumn{2}{|l|}{$\begin{array}{l}\text { Must fulfill criteria for B1a. Postpradial distress syndrome and/or B1b. } \\
\text { Epigastric pain syndrome. }\end{array}$} \\
\hline \multicolumn{2}{|l|}{$\begin{array}{l}\text { Criteria fulfilled for the last } 3 \text { months with symptom onset at least } \\
6 \text { months before diagnosis. }\end{array}$} \\
\hline B1a. Postprandial distress syndrome & B1a. Diagnostic criteria for postprandial distress syndrome \\
\hline Must include one or both of the following at least 3 days per week: & Must include one or both of the following: \\
\hline $\begin{array}{l}\text { 1. Bothersome postprandial fullness (ie, severe enough to impact } \\
\text { on usual activities) }\end{array}$ & $\begin{array}{l}\text { 1. Bothersome postprandial fullness, occurring after ordinary sized } \\
\text { meals, at least several times per week }\end{array}$ \\
\hline $\begin{array}{l}\text { 2. Bothersome early satiation (ie, severe enough to prevent finishing a } \\
\text { regular-size meal) }\end{array}$ & $\begin{array}{l}\text { 2. Early satiation that prevents finishing a regular meal, at least several } \\
\text { times per week }\end{array}$ \\
\hline $\begin{array}{l}\text { No evidence of organic, systemic, or metabolic disease that is likely to } \\
\text { explain the symptoms on routine investigations (including at upper } \\
\text { endoscopy) }\end{array}$ & $\begin{array}{l}\text { Supportive criteria } \\
\text { 1. Upper abdominal bloating or postprandial nausea or excessive } \\
\text { belching can be present }\end{array}$ \\
\hline $\begin{array}{l}\text { Criteria fulfilled for the last } 3 \text { months with symptom onset at least } \\
6 \text { months before diagnosis. }\end{array}$ & 2. Epigastric pain syndrome may coexist \\
\hline \multicolumn{2}{|l|}{ Supportive remarks } \\
\hline \multicolumn{2}{|l|}{$\begin{array}{l}\text { - Postprandial epigastric pain or burning, epigastric bloating, excessive } \\
\text { belching, and nausea can also be present }\end{array}$} \\
\hline \multicolumn{2}{|l|}{ - Vomiting warrants consideration of another disorder } \\
\hline \multicolumn{2}{|l|}{. Heartburn is not a dyspeptic symptom but may often coexist } \\
\hline \multicolumn{2}{|l|}{$\begin{array}{l}\text { Symptoms that are relieved by evacuation of feces or gas should } \\
\text { generally not be considered as part of dyspepsia }\end{array}$} \\
\hline $\begin{array}{l}\text { Other individual digestive symptoms or groups of symptoms, eg, from } \\
\text { gastroesophageal reflux disease and the irritable bowel syndrome may } \\
\text { coexist with PDS }\end{array}$ & \\
\hline
\end{tabular}


Table. Continued

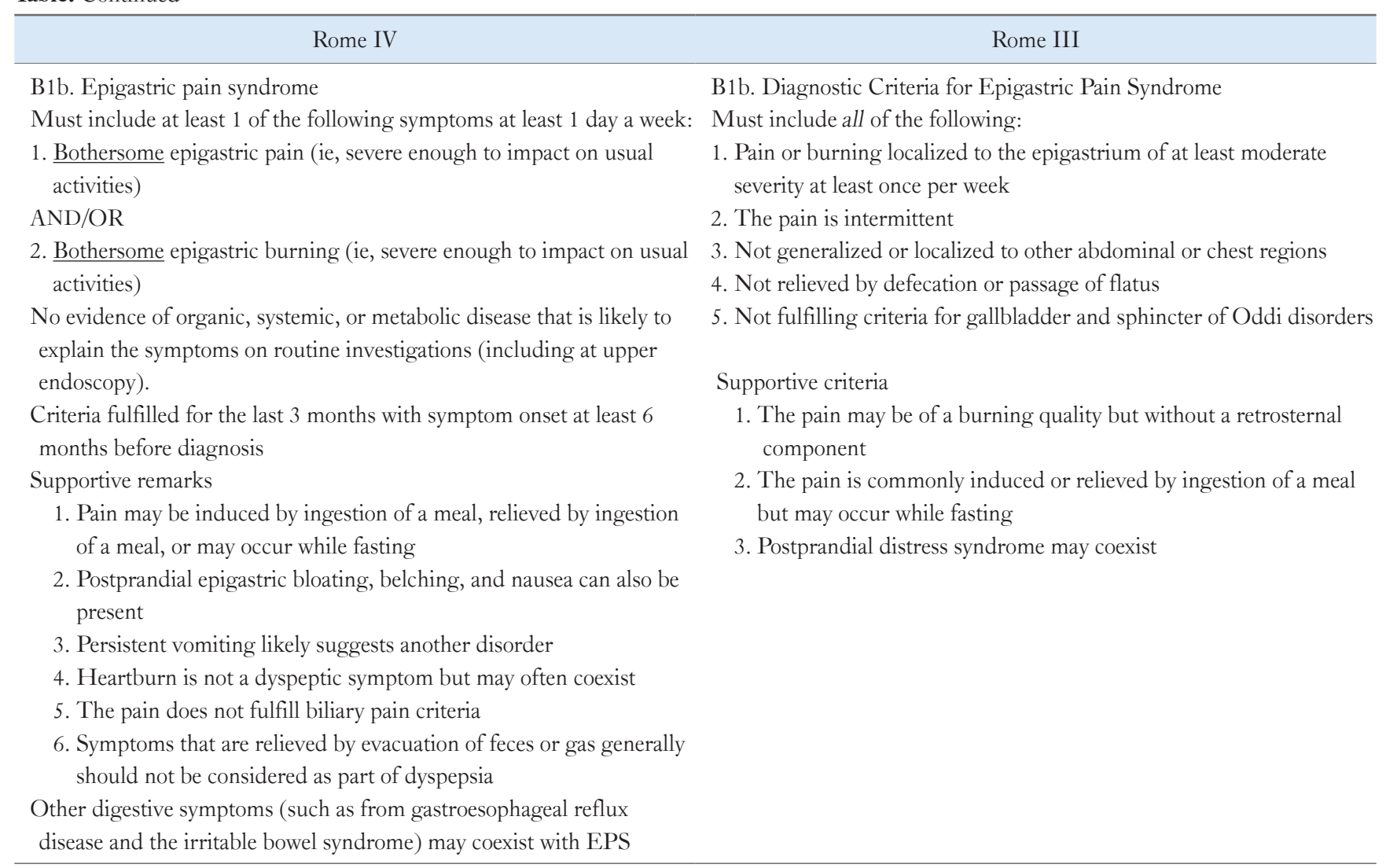

New terms in Rome IV are underlined.

and EPS. PDS has meal-induced dyspeptic symptoms, whereas EPS does not only occur postprandially. However, these two can overlap. ${ }^{12}$ Investigation on the effect of meal ingestion on symptom generation has indicated that in patients with dyspepsia, not only postprandial fullness and early satiation, but also epigastric pain or epigastric burning sensation and nausea (not vomiting) may increase after the meals. Carbone et $\mathrm{al}^{13}$ in Belgium reported that by accounting the relationship between epigastric pain and nausea in relation to meal ingestion, symptoms of PDS and EPS frequently coexist in patients with FD, and then postprandial symptoms substantially contributes to the overlap in the previous Rome III definition. Then they demonstrated that a more rigorous link of symptoms after meals to PDS might improve the separation efficiency of PDS from EPS. ${ }^{13}$ Accordingly, the definition of PDS was slightly revised by including that, in addition to postprandial fullness and early satiety that is known to occur postprandially, other symptoms such as epigastric pain and burning can be occurred or modified also by ingesting a meal (Table). ${ }^{4}$ In addition, epigastric bloating, belching, and nausea can be present in both PDS and EPS as pos- sible adjunctive features of both, while vomiting is unusual, and should prompt a search for other diagnoses such as nausea and vomiting disorder. Thus, the Rome IV criteria include not only PDS and EPS, but also PDS and EPS overlap syndromes.

The overlap of PDS and EPS in the hospital-based population is more frequent than in the general population. ${ }^{14}$ According to the population-based, cross-sectional survey study in Olmsted County by Choung et al, ${ }^{15}$ while the prevalence of dyspepsia was $15 \%$, the overlap of the FD subgroups was significantly less than expected by chance. In Korean primary and tertiary hospital data, however, the prevalences of PDS/EPS overlap were very low in both primary clinic and tertiary hospital setting $(2.8 \%$ in primary clinic and $1.9 \%$ in tertiary hospital). ${ }^{16}$ This study included the group of neither PDS nor EPS (19.4\% in primary clinic and $17.6 \%$ in tertiary hospital), in addition to PDS, EPS, and PDS/EPS overlap. ${ }^{16}$ In our web survey in $2012,{ }^{12}$ prevalence of FD was $7.0 \%$ including PDS alone $4.7 \%$ ( $67.3 \%$ of FD), EPS alone $0.8 \%$ (11.0\% of FD), and overlap of PDS and EPS $1.5 \%$ (21.7\% of FD), suggesting the less prevalence in overlap in such a population-based study in Japan. 
We also reported in 2012 the results of other web surveys in Japan ${ }^{17}$ as that prevalence of $\mathrm{FD}$ was $8.0 \%$ including PDS alone $4.8 \%$ (61.0\% of FD), EPS alone $0.8 \%$ (10.3\% of FD), and overlap of PDS and EPS 2.3\% (28.8\% of FD). On the contrary, Ghoshal and Singh ${ }^{18}$ reported a house-to-house survey in the community in India that the prevalence of functional GI disorders was $21.7 \%$ (603/2774) and dyspepsia was 14.7\% (413/2774), and that among those with dyspepsia, 9\% had EPS alone, 27\% had PDS, and 64\% EPS-PDS overlap, suggesting a relatively high rate of EPS-PDS overlap in the Indian community, different from studies of other countries. Thus, in Asia, less prevalence in overlap of PDS and EPS was shown in the survey for the web-population that can access the internet in Japan, higher prevalence in PDS and EPS overlap syndrome has been shown in population-based study in a rural community in India.

\section{Treatment of Functional Dyspepsia}

\section{A Novel and Clear Definition of Helicobacter pylori-associated Dyspepsia}

In Rome IV, Helicobacter pylori eradication therapy was described at the beginning of drug treatment. According to a systematic review and meta-analysis of clinical trials thus far, $H$. pylori eradication therapy in chronic dyspepsia patients is effective in numbers needed to treat as 15 , with a slight but statistically significant difference. ${ }^{19}$ However, this is not a treatment of FD; instead, it is the "treatment of $H$. pylori-infected dyspepsia." If symptomatic remission continues over a long period of 6 months or longer after eradication, the symptoms of dyspepsia are because of $H$. pyloriinfected gastritis and the condition is diagnosed as $H$. pyloriassociated dyspepsia, ${ }^{20,21}$ as defined by the Kyoto global consensus meeting. ${ }^{22,23}$ As described in a clinical management flow-chart of $\mathrm{FD}$ in the Rome IV edition, ${ }^{4}$ before the diagnosis of $\mathrm{FD}$ itself is made, alarm feature evaluation, upper GI endoscopy, $H$. pylori testing and treatment, and diagnosis of secondary dyspepsia as was defined by the Rome IV should be performed. ${ }^{4}$ Especially in Asia, where the prevalence of gastric cancer is high, ${ }^{24}$ prompt endoscopy ${ }^{25}$ and $H$. pylori testing and treatment would be more beneficial. ${ }^{26}$ Needless to say, eradication of $H$. pylori in dyspepsia patients is the most effective approach in terms of medical economics, as reported in young Asian uncomplicated dyspepsia patients that $H$. pylori "test and treat" in dyspepsia patients is reported to be more cost-effective than prompt endoscopy. ${ }^{27}$

\section{Anti-secretory Agents for the Treatment of Functional Dyspepsia}

Once the diagnosis of FD is determined, acid secretion inhibitors (anti-secretory agents) should be considered as the best treatment option. Even when looking at the effectiveness of PPI and $\mathrm{H}_{2} \mathrm{RA}$ against $\mathrm{FD}$, we cannot exclude the effects of overlap of reflux diseases. However, since PPI and $\mathrm{H}_{2} \mathrm{RA}$ outperformed placebo by $10-15 \%$ in many clinical trials, they are considered effective for treating FD. However, PPI is not effective in ameliorating symptoms of PDS, and this result was similar to those of our past clinical trials, which indicated that a low dose (15 mg) of lansoprazole ameliorates dyspeptic symptoms, especially the EPS-related symptoms of $\mathrm{FD}^{28}$

\section{Prokinetics}

Although prokinetics have been shown to allow a relative risk reduction of $33 \%$ compared with placebo in numbers needed to treat as 6, most data are based on clinical trials for domperidone and cisapride, which have already disappeared from the market. ${ }^{29,30}$ In such cases, publication bias is also a concern. Treatment with pure prokinetics such as erythromycin, which does not induce an antiemetic action, promotes non-physiological gastric emptying by inducing faster GI motility in the postprandial phase, but it has been determined that the effect of erythromycin is weaker compared with other treatments that combine prokinetic action and anti-emetic effect. Meanwhile, itopride, a dopamine D2 receptor inhibitor which inhibits acetylcholinesterase, has few adverse events, and improves the feeling of relaxation and early satiety after meals. In addition, pyloric injection of botulinum toxin has no effect over placebos against gastroparesis and dyspepsia symptoms.

\section{Other Treatment Options for Functional Dyspepsia}

In addition to anti-secretory agents, novel drugs such as acotiamide,${ }^{31,32}$ tandospirone, ${ }^{33}$ buspirone, ${ }^{34}$ and the herbal products STW-5 and rikkunshito ${ }^{35-37}$ are newly listed in the Rome IV criteria. In Japan, only acotiamide ${ }^{38}$ is officially approved by the national insurance system for the treatment of FD. In Asia, including Japan, not only rikkunshito but many other herbal medications ${ }^{35}$ are also used.

Although we performed a double-blind, placebo-controlled, randomized controlled clinical trial for the efficacy and safety of rikkunshito on Rome III-based FD and showed that global patient assessment tended to improve by rikkunshito, ${ }^{36}$ evidence-based clinical data are still lacking in terms of such alternative medication. 
In terms of rikkunshito, we also reported that a low baseline level of plasma des-acyl ghrelin was associated with an increased treatment efficacy of rikkunshito against $\mathrm{FD} .{ }^{37}$ In this report, the lack of alcohol consumption was also clinically useful in predicting the response to rikkunshito. ${ }^{37}$ However, further scientific evidence on the use of these herbal medicines is necessary.

Psychotropic drugs such as antidepressant drugs are often used as a second line of functional GI disorder treatment, but a multicenter, randomized controlled trial that compared tricyclic antidepressants (TCA) and recent selective serotonin re-uptake inhibitors (SSRI) in North America showed that the effect of SSRI is not significantly different from that of TCA, rather it is less tolerable, whereas low-dose amitriptyline, a TCA, is more effective compared with placebo. ${ }^{39}$

In future applications for novel therapeutic development for FD, clinical studies based on the novel Rome IV criteria should be performed.

\section{Belching Disorder}

In the revised Rome IV, excessive supragastric belching and gastric belching are clearly distinguished. Because belching does not always arise from swallowing air, the disease term "aerophagia" was not used to describe excessive belching conditions in Rome IV. Recent progress in both high-resolution manometry and impedance monitoring system enables the objective differentiation of supragastric belching from gastric belching. For supragastric belching, according to the open-label study, speech therapy conducted by a wellinformed speech therapist can significantly relieve the symptoms, although appropriate studies are further needed. ${ }^{40}$

\section{Nausea and Vomiting Disorders}

Chronic nausea can be induced without association with vomiting. Vomiting in the absence of nausea might prompt a suspicion of an organic central nervous system disease. Nausea may be mealrelated or unrelated, suggesting potential pathogenic heterogeneity. Minor changes to the CVS criteria were made to indicate the observation that some adult patients report inter-episodic milder symptoms other than vomiting, and absence of vomiting for at least a week between episodes was a distinguishing feature in adults. Namely, CVS includes stereotypical episodes of vomiting in terms of onset and short, and less than a week duration. It must include at least a discrete episode in the prior and 2 episodes in the past 6 months, occurring at least 1 week part. ${ }^{5}$ It may combine with a fam- ily or past history of migraines.

Meanwhile, CHS is absolutely distinct from CVS, as it exhibits different epidemiology, such as marijuana smoking, and has a specific pathological bathing behavior (prolonged hot baths or showers) and therapy. CHS is similar with stereotypical episodic vomiting in terms of onset, duration, and frequency. CHS often occurs in males with daily cannabis use (3-5 times/day) over at least 2 years. However, in Asia, especially in Japan, where marijuana smoking is prohibited, patients in this category might be extremely few.

\section{Rumination Syndrome}

The criteria for rumination syndrome are essentially unchanged. Continuous relapsing regurgitation of recently ingested food from the mouth is the representative symptom of this syndrome. In this syndrome, regurgitation should not be preceded by retching. In the revision, effortless regurgitation, which is usually not preceded by nausea, is emphasized as a major diagnostic point. All these supportive points are added based on clinical experience, not on scientific evidence.

\section{Summary}

With the Rome IV criteria, development of new therapeutic drugs in the field of esophagogastroduodenal disorders should be further enhanced. The Rome IV criteria are applied especially for such scientific therapeutic developments including clinical trials. Meanwhile, local guidelines that fit regional status and issues should be developed for daily clinical practice. An Asian guideline for functional esophageal or gastroduodenal disorders would play a practical role for this purpose.

Financial support: This study was supported by a Grant-in-Aid for Scientific Research (B) (16H05291), MEXT Supported Program for the Strategic Research Foundation at Private Universities (S1411003), and Keio Gijuku Academic Development Funds.

Conflicts of interest: The author received scholarshipfunds for the research from Otsuka Pharmaceutical Co, Ltd, and received service honoraria from Astellas Pharm, Astrazeneca K.K., EA Pharma Co, Ltd, Mylan EPD, Otsuka Pham, Takeda Pharm, Tsumura Co. 


\section{References}

1. Drossman DA. Functional gastrointestinal disorders: history, pathophysiology, clinical features and Rome IV. Gastroenterology 2016;150:12621279, e2.

2. Drossman DA. The functional gastrointestinal disorders and the Rome III process. Gastroenterology 2006;130:1377-1390.

3. Aziz Q, Fass R, Gyawali CP, et al. Functional esophageal disorders. Gastroenterology 2016;150:1368-1379.

4. Stanghellini V, Chan FK, Hasler WL, et al. Gastroduodenal disorders. Gastroenterology 2016;150:1380-1392.

5. Arikawa Y, Nishida H, Kurasawa O, et al. Discovery of a novel pyrrole derivative 1-[5-(2-fluorophenyl)-1-(pyridin-3-ylsulfonyl)-1H-pyrrol3-yl]-N-methylmethanamine fumarate (TAK-438) as a potassiumcompetitive acid blocker (P-CAB). J Med Chem 2012;55:4446-4456.

6. Garnock-Jones KP. Vonoprazan: first global approval. Drugs 2015;75:439-443.

7. Miwa H, Kondo T, Oshima T. Gastroesophageal reflux disease-related and functional heartburn: pathophysiology and treatment. Curr Opin Gastroenterol 2016;32:344-352.

8. Fass R, Dickman R. Non-cardiac chest pain: an update. Neurogastroenterol Motil 2006;18:408-417.

9. Galmiche JP, Clouse RE, Bálint A, et al. Functional esophageal disorders. Gastroenterology 2006;130:1459-1465.

10. Tamura Y, Funaki Y, Izawa S, et al. Pathophysiology of functional heartburn based on Rome III criteria in Japanese patients. World J Gastroenterol 2015;21:5009-5016.

11. Tack J, Talley NJ, Camilleri M, et al. Functional gastroduodenal disorders. Gastroenterology 2006;130:1466-1479.

12. Matsuzaki J, Suzuki H, Asakura K, et al. Classification of functional dyspepsia based on concomitant bowel symptoms. Neurogastroenterol Motil 2012;24:325-e164.

13. Carbone F, Holvoet L, Tack J. Rome III functional dyspepsia subdivision in PDS and EPS: recognizing postprandial symptoms reduces overlap. Neurogastroenterol Motil 2015;27:1069-1074.

14. Tack J, Talley NJ. Functional dyspepsia--symptoms, definitions and validity of the Rome III criteria. Nat Rev Gastroenterol Hepatol 2013;10:134141.

15. Choung RS, Locke GR, Schleck CD, Zinsmeister AR, Talley NJ. Do distinct dyspepsia subgroups exist in the community? A population-based study. Am J Gastroenterol 2007;102:1983-1989.

16. Park JM, Choi MG, Cho YK, et al. Functional gastrointestinal disorders diagnosed by Rome III questionnaire in Korea. J Neurogastroenterol Motil 2011;17:279-286.

17. Matsuzaki J, Suzuki H, Fukushima Y, et al. High frequency of overlap between functional dyspepsia and overactive bladder. Neurogastroenterol Motil 2012;24:821-827.

18. Ghoshal UC, Singh R. Frequency and risk factors of functional gastrointestinal disorders in a rural Indian population. J Gastroenterol Hepatol 2017;32:378-387.

19. Zhao B, Zhao J, Cheng WF, et al. Efficacy of Helicobacter pylori eradication therapy on functional dyspepsia: a meta-analysis of randomized controlled studies with 12-month follow-up. J Clin Gastroenterol 2014;48:241-247.

20. Suzuki H, Matsuzaki J, Hibi T. What is the difference between Helicobacter pylori-associated dyspepsia and functional dyspepsia? J Neurogastroenterol Motil 2011;17:124-130.

21. Suzuki H, Nishizawa T, Hibi T. Can Helicobacter pylori-associated dyspepsia be categorized as functional dyspepsia? J Gastroenterol Hepatol 2011;26(suppl 3):42-45.

22. Sugano K, Tack J, Kuipers EJ, et al. Kyoto global consensus report on Helicobacter pylori gastritis. Gut 2015;64:1353-1367.

23. Suzuki H, Mori H. Helicobacter pylori: Helicobacter pylori gastritis--a novel distinct disease entity. Nat Rev Gastroenterol Hepatol 2015;12:556557.

24. Suzuki H, Iwasaki E, Hibi T. Helicobacter pylori and gastric cancer. Gastric Cancer 2009;12:79-87.

25. Chen SL, Gwee KA, Lee JS, et al. Systematic review with meta-analysis: prompt endoscopy as the initial management strategy for uninvestigated dyspepsia in Asia. Aliment Pharmacol Ther 2015;41:239-252.

26. Miwa $\mathrm{H}$, Kusano M, Arisawa T, et al. Evidence-based clinical practice guidelines for functional dyspepsia. J Gastroenterol 2015;50:125-139.

27. Mahadeva S, Chia YC, Vinothini A, et al. Cost-effectiveness of and satisfaction with a Helicobacter pylori "test and treat" strategy compared with prompt endoscopy in young Asians with dyspepsia. Gut 2008;57:12141220.

28. Suzuki H, Kusunoki H, Kamiya T, et al. Effect of lansoprazole on the epigastric symptoms of functional dyspepsia (ELF study): a multicentre, prospective, randomized, double-blind, placebo-controlled clinical trial. United European Gastroenterol J 2013;1:445-452.

29. Moayyedi P, Soo S, Deeks J, Dlaney B, Innes M, Forman D. Pharmacological interventions for non-ulcer dyspepsia. Cochrane Database Syst Rev 2006;18:CD001960.

30. Talley NJ, Vakil NB, Moayyedi P. American gastroenterological association technical review on the evaluation of dyspepsia. Gastroenterology 2005;129:1756-1780.

31. Matsueda K, Hongo M, Tack J, Saito Y, Kato H. A placebo-controlled trial of acotiamide for meal-related symptoms of functional dyspepsia. Gut 2012;61:821-828.

32. Matsueda K, Hongo M, Ushijima S, Akiho H. A long-term study of acotiamide in patients with functional dyspepsia: results from an openlabel phase III trial in Japan on efficacy, safety and pattern of administration. Digestion 2011;84:261-268.

33. Miwa $\mathrm{H}$, Nagahara A, Tominaga K, et al. Efficacy of the $5-\mathrm{HT}_{1 \mathrm{~A}}$ agonist tandospirone citrate in improving symptoms of patients with functional dyspepsia: a randomized controlled trial. Am J Gastroenterol 2009;104:2779-2787.

34. Tack J, Janssen P, Masaoka T, Farré R, Van Oudenhove L. Efficacy of buspirone, a fundus-relaxing drug, in patients with functional dyspepsia. Clin Gastroenterol Hepatol 2012;10:1239-1245.

35. Suzuki H, Inadomi JM, Hibi T. Japanese herbal medicine in functional gastrointestinal disorders. Neurogastroenterol Motil 2009;21:688-696.

36. Suzuki H, Matsuzaki J, Fukushima Y, et al. Randomized clinical trial: 
rikkunshito in the treatment of functional dyspepsia--a multicenter, double-blind, randomized, placebo-controlled study. Neurogastroenterol Motil 2014;26:950-961.

37. Togawa K, Matsuzaki J, Kobayakawa M, et al. Association of baseline plasma des-acyl ghrelin level with the response to rikkunshito in patients with functional dyspepsia. J Gastroenterol Hepatol 2016;31:334-341.

38. Ueda M, Iwasaki E, Suzuki H. Profile of acotiamide in the treatment of functional dyspepsia. Clin Exp Gastroenterol 2016;9:83-88.

39. Talley NJ, Locke GR, Saito YA, et al. Effect of amitriptyline and escitalopram on functional dyspepsia: a multicenter, randomized controlled study. Gastroenterology 2015;149:340-349, e2.

40. Hemmink GJ, Ten Cate L, Bredenoord AJ, Timmer R, Weusten BL, Smout AJ. Speech therapy in patients with excessive supragastric belching--a pilot study. Neurogastroenterol Motil 2010;22:24-28, e2-e3. 